\title{
Inverse Compensation for Ferromagnetic Hysteresis
}

\author{
Ralph C. Smith \\ Center for Research in Scientific Computation \\ North Carolina State University \\ Raleigh, NC 27695 \\ rsmith@eos.ncsu.edu
}

\author{
Rick Zrostlik \\ Etrema Products, Inc. \\ 2500 North Loop Drive \\ Ames, IA 50010 \\ rick.zrostlik@etrema-usa.com
}

\begin{abstract}
This paper addresses the validation of an energybased inverse compensator for hysteresis in ferromagnetic transducers. At moderate to high drive levels, such transducers exhibit significant constitutive nonlinearities and hysteresis due to domain mechanisms inherent to the constituent materials. This behavior must be accommodated to utilize the full capabilities of the transducers in high performance applications. In this paper, an ODE model based on magnetostatic energy principles is employed to characterize the hysteresis and nonlinear behavior of the materials. A complementary ODE models the inverse behavior of the actuator and is employed to construct a filter which compensates for the hysteresis and permits linear control design. The capability of the model and its inverse to compensate for hysteresis in a magnetostrictive actuator is illustrated through a comparison of the input to the inverse filter with the actuator output.
\end{abstract}

\section{Introduction}

Ferromagnetic transducers provide the capability for generating the large forces and strains required in a variety of automotive, industrial and aerospace applications. While Terfenol-D is one of the more widely used ferromagnetic core materials, the list of materials currently employed for actuator design is large and growing. All of these materials exhibit a certain degree of hysteresis and nonlinear constitutive behavior at the high drive levels where the materials are advantageous (see Figure 1). In this paper, we consider the experimental validation of a model and its inverse which can be used to compensate for the hysteresis and nonlinear constitutive behavior.

To illustrate the goal in developing an appropriate inverse compensator, consider the prototypical plant depicted in Figure 2. The input to the actuator is denoted by $v$ while the input which the actuator provides to the plant is denoted by $u$. Finally, we let $u_{d}$ denote the input specified to obtain the control objective in the absence of the nonlinearities and hysteresis
$N(\cdot)$. Under moderate to high drive conditions, the map $N(\cdot)$ contains sufficient hysteresis to degrade and in some cases, destabilize linear control methods as illustrated in [8]. To permit linear control design through inverse compensation techniques, the desired input is filtered through an inverse map $N^{-1}(\cdot)$ or approximate map $\hat{N}^{-1}(\cdot)$ before input to the actuator as depicted in Figure 2b. In this manner, one compensates for the hysteresis and nonlinearities, and the final signal $u=N\left(N^{-1}\left(u_{d}\right)\right)$ is that prescribed by the control law.

This technique for accommodating actuator and sensor hysteresis and nonlinearities has been employed in a variety of settings, and the reader is referred to $[12,13]$ for a general development of the theory in the context of adaptive control design. The models employed in [12] to characterize the hysteresis $N(\cdot)$ and inverse $N^{-1}(\cdot)$ are based on piecewise linear Preisach approximations which are phenomenological in nature. The model employed in this paper is based on magnetostatic energy principles which facilitates the construction of the corresponding filter and provides the compensator with a high degree of robustness with regard to changing operating conditions.

To motivate the theory used to model the nonlinearities and hysteresis, we briefly describe the physical mechanisms which produce these phenomena. Below their Curie temperatures, all ferromagnetic materials exhibit a domain structure which consists of regions where moments are highly aligned (domains) separated by transition regions termed domain walls. Hysteresis of the type depicted in Figure 1 is typically attributed to the restriction of moment rotation and domain wall movement by inclusions or pinning sites inherent to the materials. This produces both reversible and irreversible components to the magnetization and yields the energy losses associated with Figure 1.

The irreversible and reversible domain processes are modeled using the theory of Jiles and Atherton [6, 7]. This quantifies the nonlinear and hysteretic relation between the input field and the resulting magnetization. The extension of this theory to Terfenol-D transducers was considered in $[1,2,3,4]$ and an inverse compensator 
for Terfenol-D transducers was developed in [9]. This paper presents experimental validation of that theory.

The modeling techniques used to quantify the hysteresis and nonlinear constitutive behavior are summarized in Section 2 while the model inverse is outlined in Section 3. The experimental validation of the inverse is detailed in Section 4 for a Terfenol-D magnetostrictive transducer. There it is illustrated that by filtering a desired signal $u_{d}$ by an appropriate inverse $N^{-1}(\cdot)$ before input to the transducer, one obtains an input $u$ to the plant which accurately approximates $u_{d}$. Hence the inverse can be used to compensate for actuator nonlinearities in a manner which facilitates linear control design.

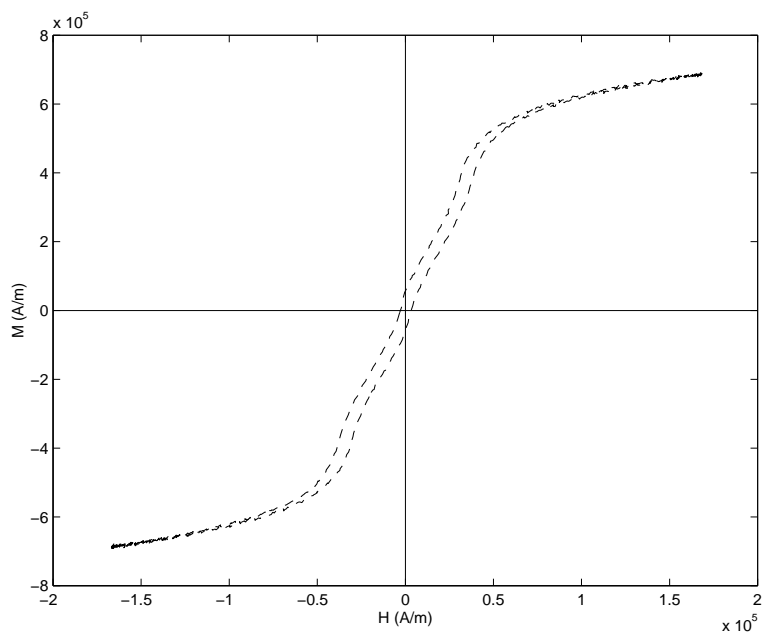

Figure 1. Relationship between the magnetic field $H$ and magnetization $M$ for Terfenol-D transducer data.

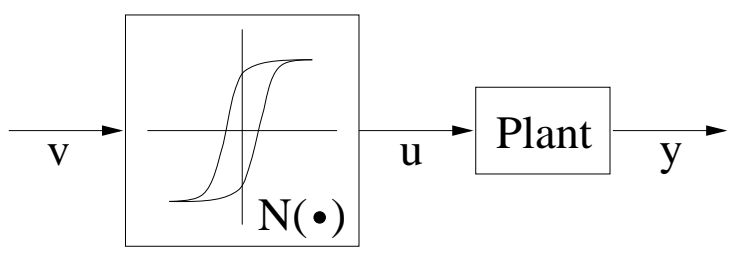

(a)

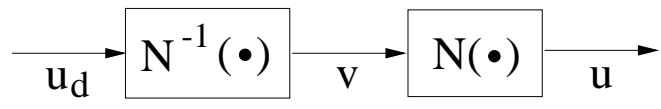

(b)

Figure 2. (a) Plant with actuator hysteresis $N(\cdot)$; (b) Inverse compensator $N^{-1}(\cdot)$ for the hysteresis.

\section{Magnetic Hysteresis Model}

The hysteresis model is based on the theory of Jiles and Atherton [6, 7] employed in the context of magnetostrictive transducers $[1,2,3]$. This theory is based on the tenant that ferromagnetic hysteresis, of the form depicted in Figure 1, is due to the impediment of domain wall movement by inclusions inherent to the materials. As detailed in [5], the formation of domain walls at inclusions or pinning sites is energetically favorable and provides a minimum energy state for the material. For low field inputs, domain wall motion is reversible and contributes a magnetization $M_{r e v}$. At higher input levels, however, the domain walls intersect remote pinning sites which corresponds to moving from one potential energy well to an adjacent well. This yields an irreversible component to the magnetization $M_{i r r}$ which is summed with the reversible component to obtain the total magnetization.

To model the reversible and irreversible effects, we consider first the hysteresis free, or anhysteretic, magnetization which would result in the absence of pinning sites. Under the assumption of constant stress $\sigma_{0}$ and isothermal operating conditions, two commonly employed models for the anhysteretic magnetization are the Langevin expression

$$
M_{a n}=M_{s}\left[\operatorname{coth}\left(\frac{H_{e f f}}{a}\right)-\left(\frac{a}{H_{e f f}}\right)\right]
$$

and the Ising spin relation

$$
M_{a n}=M_{s} \tanh \left(\frac{H_{e f f}}{a}\right)
$$

(see $[5,6])$. The effective field given by

$$
H_{\text {eff }}=H+\alpha M
$$

incorporates both the applied field $H$ and interdomain coupling and magnetoelastic interactions through the coefficient $\alpha$ and magnetization $M$. Both models are derived by quantifying the probability of dipole moments occupying various energy states through the application of Boltzmann statistics. The higher-order differences between the models is due to the underlying assumptions concerning possible cell orientations. The parameters $\alpha$ and $a$ are estimated for a given transducer through a least squares fit to the data.

As detailed in [6], the irreversible magnetization is quantified by determining the energy required to reorient dipoles in the presence of an applied field. This yields the differential equation

$$
\frac{d M_{i r r}}{d H}=\widetilde{\delta} \frac{M_{a n}-M_{i r r}}{k \delta-\alpha\left(M_{a n}-M_{i r r}\right)}
$$

where the parameter $k$ provides an average measure of the energy required to re-orient domains and hence 
translate domain walls. The parameter $\delta$ is defined to have the value +1 when $d H>0$ and -1 when $d H<0$ to guarantee that pinning always opposes changes in magnetization. In applications, $\delta$ can be directly determined from the magnetic field data. Finally, the parameter $\widetilde{\delta}$ defined by

$$
\widetilde{\delta}=\left\{\begin{array}{c}
0,\left\{d H>0 \text { and } M>M_{a n}\right\} \\
\quad \text { or }\left\{d H<0 \text { and } M<M_{a n}\right\} \\
1, \text { otherwise } .
\end{array}\right.
$$

incorporates the physical observation that following field reversal at the loop tip, the changes in magnetization are reversible until the anhysteretic value is reached [7].

The second component of the magnetization is the reversible magnetization which reflects the degree to which domain walls bend before attaining the energy necessary for translation. As derived in [6], to a first approximation, the reversible magnetization is given by

$$
M_{r e v}=c\left(M_{a n}-M_{i r r}\right) .
$$

The reversibility coefficient $c$ can be estimated from the ratio of the initial and anhysteretic differential susceptibilities [7] or through a least squares fit to data.

The total magnetization is then given by

$$
M=M_{r e v}+M_{i r r}
$$

with $M_{i r r}$ and $M_{r e v}$ defined by (3) and (4) and the anhysteretic magnetization given by (1) or (2). To provide a relation which facilitates inversion, it is advantageous to express the output magnetization as a function of the input field. When the Ising spin model is used to characterize the anhysteretic magnetization, the expressions $(2),(3)$ and (4) can be consolidated to yield

$$
\begin{aligned}
\frac{d M}{d H}= & (1-c) \frac{d M_{i r r}}{d H}+c \frac{d M_{a n}}{d H} \\
= & \widetilde{\delta}(1-c) \frac{M_{a n}-M_{i r r}}{k \delta-\alpha\left(M_{a n}-M_{i r r}\right)}+c \frac{d M_{a n}}{d H} \\
= & \frac{\widetilde{\delta}\left[M_{s} \tanh \left(\frac{H+\alpha M}{a}\right)-M\right]}{k \delta-\tilde{\alpha}\left[M_{s} \tanh \left(\frac{H+\alpha M}{a}\right)-M\right]} \\
& \quad+\frac{c M_{s}}{a} \operatorname{sech}^{2}\left(\frac{H+\alpha M}{a}\right)\left(1+\alpha \frac{d M}{d H}\right)
\end{aligned}
$$

where $\tilde{\alpha}=\frac{\alpha}{1-c}$. The magnetization at a given field level is then specified by the solution to the differential equation

$$
\begin{aligned}
& \frac{d M}{d H}=\mathcal{F}(H, M) \\
& M\left(H_{0}\right)=M_{0}
\end{aligned}
$$

where

$$
\begin{aligned}
\mathcal{F}(H, M)= & \frac{1}{1-\frac{c M_{s} \alpha}{a} \operatorname{sech}^{2}\left(\frac{H+\alpha M}{a}\right)} \\
\bullet & \left\{\frac{\widetilde{\delta}\left[M_{s} \tanh \left(\frac{H+\alpha M}{a}\right)-M\right]}{k \delta-\tilde{\alpha}\left[M_{s} \tanh \left(\frac{H+\alpha M}{a}\right)-M\right]}\right. \\
& \left.+\frac{c M_{s}}{a} \operatorname{sech}^{2}\left(\frac{H+\alpha M}{a}\right)\right\} .
\end{aligned}
$$

If one employs the Langevin expression (1) rather than the Ising spin relation (2) for the anhysteretic, the function $\mathcal{F}$ is given by

$$
\begin{aligned}
\mathcal{F}(H, M)= & \frac{1}{1+c M_{s} \alpha \frac{\partial \mathcal{L}}{\partial H}\left(\frac{H+\alpha M}{a}\right)} \\
\bullet & \left\{\frac{\widetilde{\delta}\left[M_{s} \mathcal{L}\left(\frac{H+\alpha M}{a}\right)-M\right]}{k \delta-\tilde{\alpha}\left[M_{s} \mathcal{L}\left(\frac{H+\alpha M}{a}\right)-M\right]}\right. \\
& \left.-c M_{s} \frac{\partial \mathcal{L}}{\partial H}\left(\frac{H+\alpha M}{a}\right)\right\}
\end{aligned}
$$

where the Langevin function is defined by

$$
\mathcal{L}(z) \equiv \operatorname{coth}(z)-\frac{1}{z}
$$

The relation (6) with $\mathcal{F}$ given by (7) or (8) is then employed when quantifying the actuator hysteresis $N(\cdot)$. In both cases, the parameters $\delta$ and $\widetilde{\delta}$ can be computed directly from the measured field data or the computed values of the magnetization and anhysteretic magnetization. The saturation magnetization $M_{s}$ can often be determined directly from the measured data or a priori knowledge of the material behavior. Hence only the parameters $a, \alpha, c$ and $k$ must be determined through a least squares fit to data or adaptive parameter estimation techniques.

Finally, we note that the time-dependent dynamics of the magnetization can be specified through the chain rule

$$
\frac{d M}{d t}=\mathcal{F}(H, M) \frac{d H}{d t} .
$$

This relation should be employed only for quasistatic processes, however, since the model (6) neglects the elastic properties of the material as well as timedependent loss mechanisms.

\section{Inverse Hysteresis Model}

The hysteresis model in the form (6) is amenable to inversion through consideration of a complementary differential equation. For actuators in which the anhysteretic is modeled by the Ising spin model, the inverse 
is specified through solution of the differential equation

$$
\begin{aligned}
& \frac{d M^{-1}}{d H}=\frac{1}{\mathcal{F}\left(M^{-1}, H\right)} \\
& M^{-1}\left(H_{0}\right)=M_{0}^{-1}
\end{aligned}
$$

with $\mathcal{F}$ defined by (7). Similarly, employment of (8) provides the inverse when the Langevin model is used to characterize the anhysteretic magnetization.

In the control nomenclature of Section 1, the definition (9) can be combined with passive actuator or structural components to provide the exact inverse $N^{-1}(\cdot)$ if the parameters $M_{s}, \alpha, a, c$ and $k$ are known exactly. For the examples in this paper, it is assumed that suitable parameters have been obtained through a least squares fit to data and the exact inverse is employed. Similarly it provides an approximate inverse $\widehat{N}^{-1}(\cdot)$ which is suitable for adaptive parameter estimation and control methods if the parameters are unknown or slowly change due to changing operating conditions. Details regarding the design of control methods which employ an approximate inverse can be found in [12].

\section{Experimental Validation}

To illustrate the performance of the model and inversion technique, we consider the characterization and hysteresis compensation for a prototypical magnetostrictive transducer employed at Etrema Products. As depicted in Figure 3, the primary components in the transducer are the driving Terfenol-D rod, a wound wire solenoid which surrounds the rod, and a prestress mechanism comprised of Belleville washers which maintain the rod in a state of compression. The results reported here were obtained with a prestress of approximately $\sigma_{0}=1.0 \mathrm{ksi}$. The Terfenol-D $\left(\mathrm{Tb}_{0.3} \mathrm{Dy}_{0.7} \mathrm{Fe}_{1.92}\right) \mathrm{rod}$ had a length of $0.254 \mathrm{~m}$, a diameter of $0.020 \mathrm{~m}$, and was composed of four laminae to minimize eddy current losses. The drive solenoid was constructed from 14 AWG round wire with an air gap left between the coil and Terfenol rod to permit cooling. Additionally, a pickup coil comprised of 34 AWG wire was wrapped directly around the rod for sensing purposes.

For characterization and validation of the inverse compensator, it was necessary to measure the field $H$ and magnetization $M$ produced in the transducer. The current $I(t)$ was used to compute the field using the relation $H(t)=n I(t)$ where $n$ is the number of turns in the drive coil per unit length. We note that this relation ignores end effects in the rod and the reader is referred to $[2,3]$ for details concerning the construction of an empirical filter which incorporates the spatial behavior of the rod. From the induced voltage in the pickup coil, the Faraday-Lenz law was used to compute the temporal derivative of the magnetic induction $B$. Integration then yielded the induction and magnetiza- tion $M=\frac{1}{\mu_{0}} B-H$. Finally, we note that the data was collected at $1 \mathrm{~Hz}$.

The first step in the construction of the inverse compensator was the determination of parameters $a, \alpha, k, c$ and $M_{s}$ so that the model accurately characterizes the transducer. While initial values for the parameters can be directly determined from measured values of the differential susceptibility and field [7], or a priori manufacturer specifications, final values are typically determined through a least squares fit to the data.

The model response obtained with the parameters $M_{s}=7.65 \times 10^{5} \mathrm{~A} / \mathrm{m}, a=1.5 \times 10^{5} \mathrm{~A} / \mathrm{m}, \alpha=0.032$, $k=5000 \mathrm{~A} / \mathrm{m}$, and $c=0.18$ is compared with the data in Figure 4. We note that while the data exhibits changes of concavity in the burst region which are not incorporated in the model, the model accurately quantifies the hysteresis and saturation behavior of the transducer.

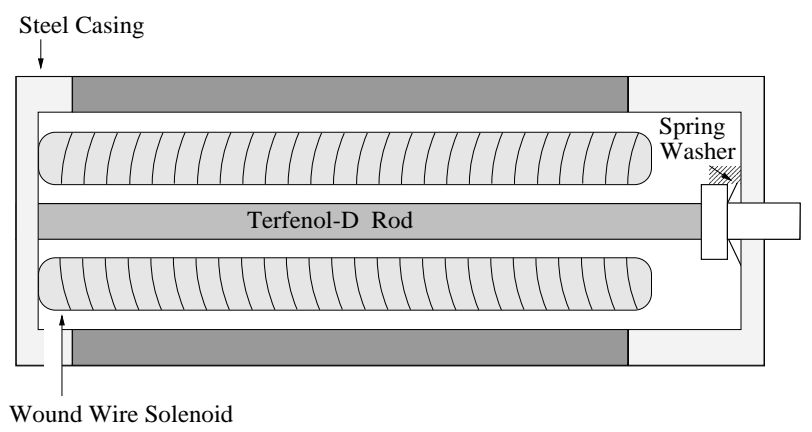

Figure 3. Cross section of the Terfenol-D magnetostrictive transducer.

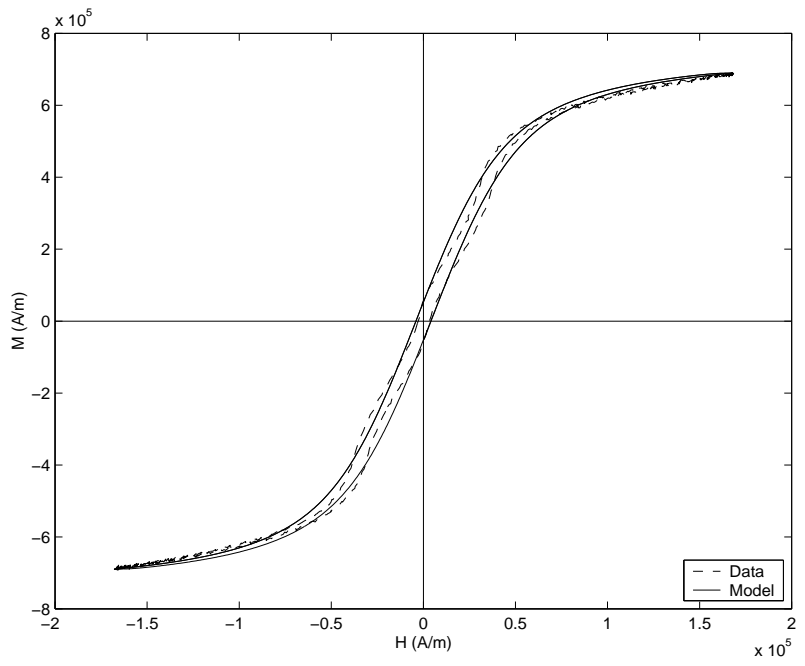

Figure 4. Experimental $M-H$ data $(---)$ and model fit $(-)$. 
The inverse (9) with the above parameter values was then employed as a filter for the physical transducer in the manner illustrated in Figure 2. A $1 \mathrm{~Hz}$ triangular input $u_{d}$ was applied to the inverse to generate a signal $v$ which was input to the transducer. The output $u$ from the transducer was then measured to determine the accuracy of the compensation technique.

The input signal $u_{d}$ is compared with the transducer response $u$ in Figure 5. It is observed that while $u$ exhibits slight deviations due to unmodeled domain rotation effects in the burst region, the overall fit is accurate. Hence the inverse provided by this model adequately compensates for the hysteresis and saturation behavior of the transducer.

From the perspective of control design, it is necessary to quantify the degree to which the transducer can accommodate various inputs. Aspects of this question are illustrated in Figure 6. Here the field $v$ prescribed by the inverse compensator is compared with the field which is actually measured in the transducer. The two agree through the low and middle drive ranges but the measured field does not attain the peak values due to limitations in the hardware and amplifier. This can be alleviated by limiting the rate and magnitude of prescribed inputs.

\section{Concluding Remarks}

The results presented here illustrate both the performance of a domain wall model for quantifying hysteresis and nonlinear constitutive behavior in a magnetostrictive transducer and the development of an inverse compensator based on this model. The model and its inverse are formulated as ODE which are implemented using low-order approximation techniques. The efficiency of the method is further augmented by the fact that the model and inverse rely on only five parameters, some of which can be directly approximated from a priori specifications or measured properties of the data.

A comparison of the input $u_{d}$ to the model inverse with the output data $u$ from the physical transducer illustrates that the technique accurately compensates for the hysteresis and nonlinear behavior of the transducer. Furthermore, the observation that unmodeled phenomena are not exacerbated by the inverse hints at the stability of the technique.

The design of linear control methods which employ inverse compensators has been well established in the literature (e.g., see [12]) and initial numerical investigations have indicated the viability of LQR designs, for structures employing magnetostrictive transducers, using this compensation technique [9]. Current investigations are focussed on the experimental implementation of linear control methods which utilize this compensator, and the extension of these techniques to ferroelectric materials $[10,11]$.

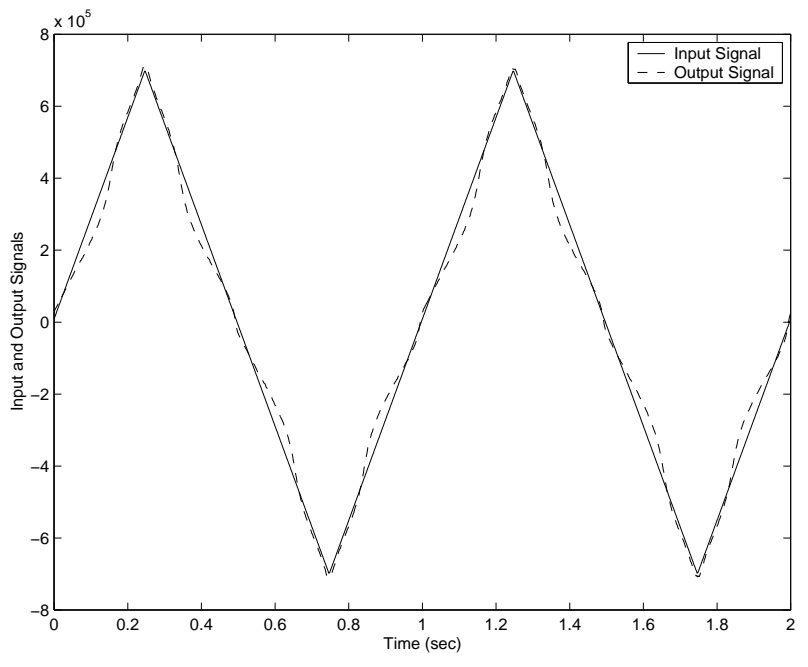

Figure 5. The input $u_{d}$ to the inverse (-) and output $u$ from the transducer $(---)$.

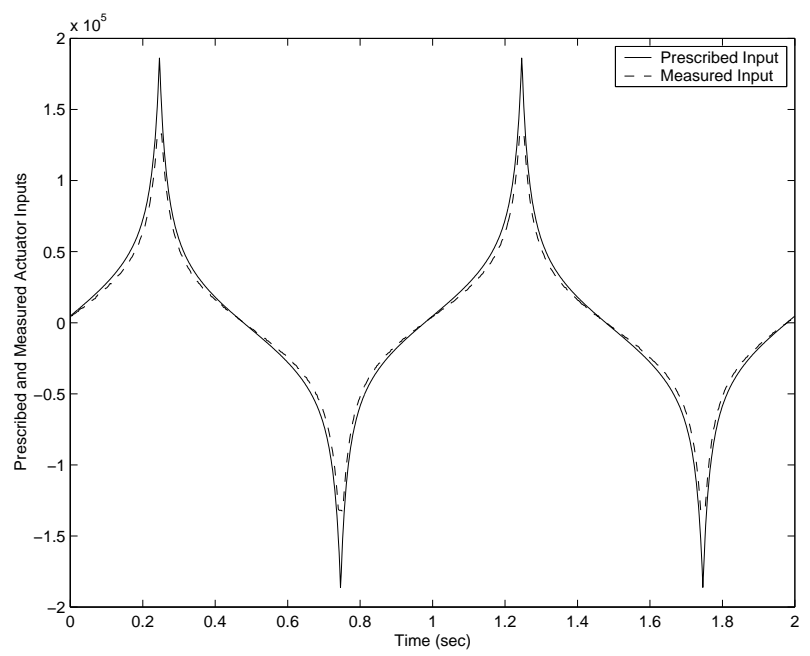

Figure 6. The field $v$ prescribed by the inverse as input to the actuator (-) and the field measured in the actuator $(---)$.

\section{Acknowledgements}

The authors express sincere appreciation to Marcelo Dapino and Julie Slaughter for input regarding the modeling techniques employed here. The research of R.C.S. was supported in part by the Air Force Office of Scientific Research under the grant AFOSR-F4962098-1-0180. 


\section{References}

[1] F.T. Calkins, R.C. Smith and A.B. Flatau, "An energy-based hysteresis model for magnetostrictive transducers," ICASE Report 97-60; IEEE Transactions on Magnetics, to appear.

[2] M.J. Dapino, R.C. Smith and A.B. Flatau, "An active and structural strain model for magnetostrictive transducers," Smart Structures and Materials 1998: Proceedings of the SPIE Conference on Smart Structures and Integrated Systems, San Diego, CA, March $1-5,1998$, pp. 198-209.

[3] M.J. Dapino, R.C. Smith and A.B. Flatau, "A structural-magnetic strain model for magnetostrictive transducers," CRSC Technical Report CRSCTR98-31; IEEE Transactions on Magnetics, to appear.

[4] M.J. Dapino, R.C. Smith and A.B. Flatau, "A coupled structural-magnetic strain model for magnetostrictive transducers," Proceedings of the SPIE Conference on Smart Structures and Integrated Systems, San Diego, CA, March 1-4, 1999.

[5] D.C. Jiles, Introduction to Magnetism and Magnetic Materials, Chapman and Hall, New York, 1991.

[6] D.C. Jiles and D.L. Atherton, "Theory of ferromagnetic hysteresis," Journal of Magnetism and Magnetic Materials, 61, 1986, pp. 48-60.

[7] D.C. Jiles, J.B. Thoelke and M.K. Devine, "Numerical determination of hysteresis parameters for the modeling of magnetic properties using the theory of ferromagnetic hysteresis," IEEE Transactions on Magnetics, 28(1), pp. 27-35, 1992.

[8] R.C. Smith, "A nonlinear optimal control method for magnetostrictive actuators," Journal of Intelligent Material Systems and Structures, 9(6), 1998, pp. $468-486$.

[9] R.C. Smith, "Inverse compensation for hysteresis in magnetostrictive transducers," CRSC Technical Report CRSC-TR98-36; Mathematical and Computer Modeling, to appear.

[10] R.C. Smith and C.L. Hom, "A domain wall model for ferroelectric hysteresis," SPIE Conference on Mathematics and Control in Smart Structures, SPIE Volume 3667, Newport Beach, CA, March 1-4, 1999, pp. $150-161$.

[11] R.C. Smith and C.L. Hom, "A domain wall theory for ferroelectric hysteresis," CRSC Technical Report CRSC-TR99-01; Journal of Intelligent Material Systems and Structures, to appear.
[12] G. Tao and P.V. Kokotović, Adaptive Control of Systems with Actuator and Sensor Nonlinearities, John Wiley and Sons, New York, 1996.

[13] B. Widrow and E. Walach, Adaptive Inverse Control, Prentice Hall, NJ, 1996. 